\title{
On real hypersurfaces of a complex projective space
}

\author{
By Yoshiaki MAEDA
}

(Received June 17, 1975)

\section{$\S 0$. Introduction.}

Let $P^{m}(C)$ denote a complex projective space equipped with the FubiniStudy metric normalized so that the maximum sectional curvature is 4 . We consider a real hypersurface $M$ of $P^{m}(C)$. It is well-known that there does not exist a totally umbilical real hypersurface of $P^{m}(C)$ (See Tashiro-Tachibana [7].) More generally, there does not exist a real hypersurface of $P^{m}(C)$ with the parallel second fundamental tensor. This is immediately seen from the Codazzi equation of the immersion of $M$. From this point of view, in this paper, we will estimate the norm of the derivative of the second fundamental tensor, and we get.

THEOREM A. Let $M$ be a complete real hypersurface of $P^{m}(C)$. Then $\|\nabla H\|^{2} \geqq 4(m-1)$, the equality holds if and only if $M$ is congruent to $M_{p, q}^{c}$ for some $p, q$.

The model space $M_{p, q}^{c}$ in the above theorem is discribed in the following.

Let $S^{2 m+1}$ be a Euclidean $(2 m+1)$-sphere of curvature 1 . We consider the Hopf fibration $\tilde{\pi}$ :

$$
S^{1} \longrightarrow S^{2 m+1} \stackrel{\tilde{\pi}}{\longrightarrow} P^{m}(C),
$$

which is the Riemannian submersion with totally geodesic fibres.

Let $\bar{M}$ and $M$ be Riemannian manifolds of dimension $2 m, 2 m-1$ respectively and $\pi: \bar{M} \rightarrow M$ be a differentiable map. $(\bar{M}, M, \pi)$ is called a Riemannian submersion compatible with the Hopf fibration $\tilde{\pi}$ if the following conditions are satisfied.

(S1) $\bar{M}$ and $M$ are (real) hypersurfaces of $S^{2 m+1}$ and $P^{m}(C)$ respectively.

(S2) $\pi: \bar{M} \rightarrow M$ is a Riemannian submersion with totally geodesic fibres such that the following diagram commutes:

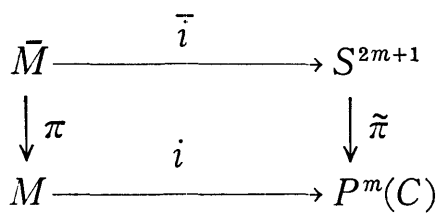

where $\bar{i}$ and $i$ denote the immersions in (S1). 
To consider a model space $M_{p, q}^{c}$ in this situation, we take a family of the products of spheres $M_{n, n^{\prime}}=S^{n} \times S^{n^{\prime}}$, where $n+n^{\prime}=2 m$. Choosing $n$ and $n^{\prime}$ to be odd, namely $n=2 p+1, n^{\prime}=2 q+1$, we put $\bar{M}=M_{2 p+1,2 q+1}$. Then we get a fibration $\pi$ :

$$
S^{1} \longrightarrow M_{2 p+1,2 q+1} \stackrel{\pi}{\longrightarrow} M_{p, q}^{c} .
$$

$\left(M_{2 p+1,2 q+1}, M_{p, q}^{c}, \pi\right)$ satisfies (S1) and (S2) (cf. [2], [3]).

$M_{p, q}^{c}$ thus obtained has a characteristic property, which can be used to prove $M$ to be congruent to $M_{p, q}^{r}$ for some $p, q$. In general, a real hypersurface $M$ of $P^{m}(C)$ has two structures, namely the contact structure induced from $P^{m}(C)$ and the submanifold structure represented by the second fundamental tensor of $M$ on $P^{m}(C)$. It might be interesting to study the relations between the two structures. In particular, for the model space $M_{p, q}^{c}$, the relation is precisely obtained through the study of the submersion $\pi$. Okumura [3] proved the following theorem which is a characterization of $M_{p, q}^{c}$.

THEOREM 0. Let $M$ be a real hypersurface of $P^{m}(C)$ and $\pi: \bar{M} \rightarrow M$ the submersion which is compatible with the Hopf fibration $\tilde{\pi}: S^{1} \rightarrow S^{2 m+1} \rightarrow P^{m}(C)$. Then the second fundamental tensor of $\bar{M}$ is parallel if and only if the contact structure of $M$ induced from $P^{m}(C)$ commutes with the second fundamental tensor of $M$.

Subsequently, a further observation on $M_{p, q}^{c}$ will be made. By use of the compatible submersion $\pi$, the hypersurface $M$ of $P^{m}(C)$ related to $\bar{M}$ has been studied in [2], [3] and [6]. Namely, Lawson[2] studied the pinching problem of the second fundamental tensor when $M$ is a minimal hypersurface of $P^{m}(C)$, and Okumura [3] also studied the pinching problem on the more general condition that the hypersurface $M$ has the constant mean curvatute.

When $\bar{M}$ is 1 ) an Einstein space or 2) a locally symmetric space, it is well known that $\bar{M}$ has parallel second fundamental tensor. Projecting the quantities on $\bar{M}$ onto $M$ in $P^{m}(C)$, we can consider the hypersurface with the conditions corresponding to 1 ) or 2). Using Theorem 0 , we will study the above hypersurfaces in $\S 4$ and $\S 5$.

The author thanks Prof. M. Obata for his valuable suggestions and encouragement during the preparation of this paper.

\section{$\S 1$. Preliminaries.}

Let $M$ be a real hypersurface of $P^{m}(C)$ and $i: M \rightarrow P^{m}(C)$ denote the isometric immersion. In a neighborhood of each point, we choose a unit normal vector field $N$ in $P^{m}(C)$. The Riemannian connections $D$ in $P^{m}(C)$ and $\nabla$ in $M$ are related by the following formulas for arbitrary vector fields $X$ and $Y$ 
on $M$ :

$$
\begin{aligned}
& D_{i_{*} X} i_{*} Y=i_{*}\left(\nabla_{X} Y\right)+g(H X, Y) N, \\
& D_{i_{*} X} N=-i_{*}(H X),
\end{aligned}
$$

where $g$ denotes the Riemannian metric induced from the Fubini-Study metric $G$ on $P^{m}(C)$, i. e., $g(X, Y)=G\left(i_{*} X, i_{*} Y\right)$, and $H$ is the second fundamental tensor of $M$ in $P^{m}(C)$.

The mean curvature $\mu$ of $M$ in $P^{m}(C)$ is defined by $\mu=$ trace $H$. If $\mu=0$, then $M$ is called a minimal hypersurface.

An eigenvector $X$ of the second fundamental tensor $H$ is called a principal curvature vector, or simply a P.C. vector. Also an eigenvalue $r$ of $H$ is called a principal curvature. In what follows, we denote $V_{r}$ the eigenspace of $H$ with eigenvalue $r$.

It is known that $M$ has an almost contact metric structure induced from the complex structure $F$ on $P^{m}(C)$, (cf. [3]), i. e., we define a tensor $f$ of type $(1,1)$, a vector field $U$ and a 1 -form $u$ on $M$ by the following:

$$
g(f X, Y)=G\left(F i_{*} X, i_{*} Y\right), \quad g(U, X)=u(X)=G\left(F i_{*} X, N\right) .
$$

Then we have

$$
f^{2} X=-X+u(X) U, \quad g(U, U)=1, \quad f U=0 .
$$

From the above remark and (1.1), we have easily

$$
\begin{aligned}
& \left(\nabla_{X} f\right) Y=u(Y) H X-g(H Y, X) U, \\
& \nabla_{Y} U=f H Y .
\end{aligned}
$$

Let $\bar{R}$ and $R$ be the curvature tensors of $P^{m}(C)$ and $M$ respectively. Since the curvature tensor $\bar{R}$ has a nice form, we have the following Gauss and Codazzi equations.

$$
\begin{aligned}
g(R(X, Y) Z, W)= & g(Y, Z) g(X, W)-g(X, Z) g(Y, W) \\
& +g(f Y, Z) g(f X, W)-g(f X, Z) g(f Y, W) \\
& -2 g(f X, Y) g(f Z, W)+g(H Y, Z) g(H X, W) \\
& -g(H X, Z) g(H Y, W)
\end{aligned}
$$

and

$$
\left(\nabla_{X} H\right) Y-\left(\nabla_{Y} H\right) X=u(X) f Y-u(Y) f X-2 g(f X, Y) U .
$$

Using (1.3), (1.6) and (1.7), we get 


$$
g\left(R_{0} X, Y\right)=(2 m+1) g(X, Y)-3 u(X) u(Y)+\mu g(H X, Y)-g\left(H^{2} X, Y\right),
$$

where $\mu=\operatorname{trace} H$ and $R_{0}$ denotes the Ricci tensor on $M$.

$$
g\left(\left(\nabla_{X} H\right) Y, U\right)-g\left(\left(\nabla_{Y} H\right) X, U\right)=-2 g(f X, Y) .
$$

\section{$\S 2$. The fundamental lemmas on a real hypersurface of $P^{m}(C)$.}

Let $M$ be a real hypersurface of $P^{m}(C)$ and assume that the trajectories of the induced vector field $U$ are geodesics, i.e.,

$$
\nabla_{U} U=0
$$

because $U$ is a unit vector. Using (1.5), (2.1) becomes

$$
f H U=0 .
$$

Applying $f$ to (2.2) and using (1.3), we get

$$
H U=\alpha U,
$$

where $\alpha=g(H U, U)$. Thus we have

LEMMA 2.1. In order that the trajectories of $U$ be geodesics, it is necessary and sufficient that $U$ be a P.C. vector.

Differentiating (2.3) covariantly along $X$ and making use of (1.4), we have

$$
g\left(\left(\nabla_{X} H\right) Y, U\right)+g(H f H X, Y)=(X \alpha) g(U, Y)+\alpha g(f H X, Y) .
$$

Making a similar equation by changing $X$ and $Y$ in the last equation and using (1.9), we get

$$
2 g(H f H X-f X, Y)=(X \alpha) u(Y)-(Y \alpha) u(X)+g((f H+H f) X, Y) .
$$

If we replace $X$ by $U$ in (2.4), we obtain

$$
Y \alpha=(X \alpha) u(Y) .
$$

Substituting (2.5) into (2.4), we have

$$
2 H f H-2 f=\alpha(H f+f H) .
$$

LEMMA 2.2. Assume that the trajectories of $U$ are geodesics. If $X$ belongs to $V_{r}$ and is orthogonal to $U$, then $f X$ belongs to $V_{(\alpha r+2) /(2 r-\alpha)}$.

Proof. From (2.6), we get for a P.C. vector $X$ which is orthogonal to $U$,

$$
(2 r-\alpha) H f X=(\alpha r+2) f X .
$$


If $2 r-\alpha=0$, then $\alpha r+2=0$. Hence we have the Lemma.

From Lemma 2.2, we easily obtain

Proposition 2.3. There exists no open set $O$ of $M$ such that at every point of $O, f H+H f=0$.

LEMMA 2.4. If the trajectories of $U$ are geodesics, then $\alpha$ is locally constant.

Proof. Since $U$, is a P.C. vector of $M$, from Lemma 2.2 we get by (2.5), $\operatorname{grad} \alpha=\beta U$, where $\beta=U \alpha$. Differentiating this equation covariantly along $X$, we have

$$
\nabla_{X} \operatorname{grad} \alpha=(X \beta) U+\beta f H X,
$$

from which, together with the fact that

$$
g\left(\nabla_{X} \operatorname{grad} \alpha, Y\right)=g\left(\nabla_{Y} \operatorname{grad} \alpha, X\right),
$$

we get

$$
(Y \beta) u(Y)-(X \beta) u(X)=\beta g((f H+H f) X, Y) .
$$

Replacing $X$ by $U$ and making use of (2.5) and (2.6), we have

$$
Y \beta=(U \beta) g(U, Y) \text {. }
$$

Substituting (2.8) into (2.7), we obtain

$$
\beta \cdot g((f H+H f) X, Y)=0 .
$$

Thus we have the lemma by Proposition 2.3.

At each point, we can take orthonormal vectors $U, X_{a}, f X_{a}(a=1, \cdots, m-1)$ which are P.C. vectors. Then any tangent vector can be expressed in the following form:

$$
X=x U+\sum_{a=1}^{m-1} x^{a} X_{a}+\sum_{a=1}^{m-1} y^{a} f X_{a}
$$

Using the above expression of $X$, we get

PROPOSITION 2.5. Let $M$ be a real hypersurface of $P^{m}(C)$ and assume that the trajectories of $U$ are geodesics. Assume that $f X$ belongs to $V_{r}$ for any $X \in V_{r}$. Then $f$ and $H$ are commutative. Furthermore by Theorem 0 , for the submersion $(\bar{M}, M, \pi)$ compatible with $\tilde{\pi}, \bar{M}$ has the parallel second fundamental tensor.

\section{§3. Proof of Theorem A.}

For a compatible submersion $(\bar{M}, M, \pi)$ with the Hopf fibration $\tilde{\pi}$, it is well known (cf. Ishihara and Konishi [1]) that if $\bar{M}$ has the parallel second 
fundamental form, $M$ satisfies

$$
g\left(\left(\nabla_{\boldsymbol{Z}} H\right) X, Y\right)=-u(X) g(f Z, Y)-u(Y) g(f Z, X) .
$$

Now, we consider the converse problem, namely we determine the hypersurface $M$ satisfying (3.1).

From (3.1) and the commutativity of the trace and the derivation, we have LEMMA 3.1. If $M$ satisfies (3.1), then the mean curvature is constant.

Using the Ricci identity, (3.1) and (1.9), we get

$$
\begin{aligned}
& g(H Y, W) g(L X, Z)+g(H Y, Z) g(L X, W)-g(H X, W) g(L Y, Z) \\
- & g(H X, Z) g(L Y, W)-g(f X, W) g(A Y, Z)-g(f X, Z) g(A Y, W) \\
+ & g(f Y, Z) g(A X, W)+g(f Y, W) g(A X, Z)-2 g(f X, Y) g(A Z, W) \\
= & 0,
\end{aligned}
$$

where $L$ and $A$ are tensor fields of type $(1,1)$ which are respectively defined by the following:

$$
\begin{aligned}
& L X=X-u(X) U-H^{2} X, \\
& A X=(f H-H f) X .
\end{aligned}
$$

Then $L$ and $A$ are symmetric linear operators. If $A=0$, then $f$ and $H$ are commutative.

Contracting (3.2) with $X$ and $W$, we have

$$
\begin{gathered}
\mu g(L Y, Z)-\left(2 m+2-\operatorname{trace} H^{2}\right) g(H Y, Z)+2 g(H Z, U) u(Y) \\
+2 g(H Y, U) u(Z)-4 g(f H f Y, Z)=0 .
\end{gathered}
$$

Replacing $Y$ by $U$ in (3.3) and using (1.3), we have

$$
\mu g\left(H^{2} X, U\right)=2 \alpha u(X)-\left(2 m-\operatorname{trace} H^{2}\right) g(H X, U),
$$

where $\alpha=g(H U, U)$.

On the other hand, replacing $X$ and $Z$ by $U$ in (3.2) and exchanging $Y$ and $W$, we get

$$
g(H Y, U) g\left(H^{2} W, U\right)=g(H W, U) g\left(H^{2} Y, U\right) .
$$

Considering (3.5), we get, for some scalar $a$,

$$
g\left(H^{2} X, U\right)=a g(H X, U),
$$

because of Schwarz's inequality.

Substituting (3.6) into (3.4), we have 


$$
b g(H X, U)=2 g(H U, U) u(X),
$$

where $b=a \mu+2 m$-trace $H^{2}$.

Lemma 3.2. For any point $p \in M, U$ is a P.C. vector.

Proof. If $b \neq 0$, then $U$ is a P.C. vector by (3.7). If $b=0$, then $g(H U, U)=0$, and we easily obtain $H U=0$ by (3.5),

We can put $H U=\alpha U$ for any point $p \in M$ because of Lemma 3.2. Then by Lemma 2.4, we see that $\alpha$ is constant.

Differentiating this equation and using (3.2), we get

$$
\alpha g(f H X, Y)=-g(f X, Y)+g(H f H X, Y) .
$$

Interchanging $X$ and $Y$ in (3.8), we have $\alpha g(A X, Y)=0$.

Now we prove

Proposition 3.3. Let $M$ be a real hypersurface of $P^{m}(C)$ satisfying (3.1). Then $f$ and $H$ are commutative.

PROOF. If $\alpha \neq 0$, it is clear from (3.8). In case $\alpha=0$, replacing $W$ by $f W$ in (3.2) and contracting $X$ and $W$, we get

$$
(2 m+2) g(A X, Y)=0 \text {. }
$$

This means $A=0$. By Theorem 0, we have

THEOREM 3.4. For a submersion $(\bar{M}, M, \pi)$ compatible with the Hopf fibration $\tilde{\pi}: S^{1} \rightarrow S^{2 m+1} \rightarrow P^{m}(C)$, the second fundamental tensor of $\bar{M}$ is parallel if and only if $M$ satisfies (3.1).

From this fact and theorems in Ryan's paper [4], we have

THEOREM 3.5. $M_{p, q}^{c}$ are only complete hypersurfaces of $P^{m}(C)$ satisfying (3.1).

Define a tensor $T$ by

$$
T(X, Y) Z=g\left(\left(\nabla_{Z} H\right) X, Y\right)+u(X) g(f Z, Y)+u(Y) g(f Z, X) .
$$

Calculating the norm of $T$ and using (1.4)] and [1.7), we get $\|\nabla H\|^{2} \geqq 4(m-1)$. Theorem $\mathrm{A}$ is thereby proved by Theorem 3.5,

\section{§4. $C$-Einstein hypersurface of $P^{m}(C)$.}

Let $M$ be a real hypersurface of $P^{m}(C)$. If the Ricci tensor $R_{0}$ of $M$ satisfies

$$
g\left(R_{0} X, Y\right)=a g(X, Y)+b u(x) u(Y),
$$

where $u$ is the induced 1 -form defined in $\S 1$, we call $M$ a $C$-Einstein hypersurface. When $b=0, M$ is an Einstein space. Now we will consider a $C$ - 
Einstein hypersurface.

We define a symmetric tensor $K$ of type $(1,1)$ by

$$
K=H^{2}-\mu H,
$$

where $H$ is the second fundamental tensor of $M$.

LEMMA 4.1. If $M$ satisfies (4.1) and $b \neq-3$ at every point of $M$, then $U$ is an eigenvector of $K$ whose eigenvalue is equal to $(2 m-2-a-b)$. Furthermore the other eigenvalues of $K$ are equal to $(2 m+1-a)$.

ProOF. By the above assumption and (1.8), we get

$$
K X=(2 m+1-a) X-(b+3) u(X) U .
$$

This equation implies the lemma.

On the other hand, at each point we can take $X_{1}, \cdots, X_{2 m-1}$ which are P.C. vectors with principal curvature $r_{1}, \cdots, r_{2 m-1}$ respectively and form as orthonormal bases. From (4.2), we get

$$
K X_{i}=\left(r_{i}^{2}-\mu r_{i}\right) X_{i}
$$

LEMMA 4.2. Under the assumptions of Lemma 4.1, $U$ is a P.C. vector whose multiplicity is equal to 1.

PROOF. (4.3) means that each $X_{i}$ is the eigenvector of $K$. Then there exists a unique vector $X$ with eigenvalue $(2 m-2-a-b)$. It follows that the eigenspace of $X$ coincides with the space of $U$. We get the lemma.

We can take an orthonormal basis $\left\{U, X_{2}, \cdots, X_{2 m-1}\right\}$ each of which is a P.C. vector with principal curvature $\alpha, r_{i}(i=2, \cdots, 2 m-1)$ respectively. From Lemma 4.1 and (4.3), we have

$$
\begin{aligned}
& r_{i}{ }^{2}-\mu r_{i}-(2 m+1-a)=0, \quad(i=2, \cdots, 2 m-1), \\
& \alpha^{2}-\mu \alpha-(2 m-2-a-b)=0 .
\end{aligned}
$$

Thus we have proved

LEMMA 4.3. Under the same assumptions as in Lemma 4.1, $M$ has at most three distinct principal curvature at each point of $M$.

On the other hand, by Lemma 2.2 we find that the only possibilities are the following cases at any point $p$ of $M$.

Case 1) $f X$ belongs to $V_{r}$ for any P.C. vector $X \in V_{r}$.

Case 2) there exists a P.C. vector $X \in V_{r}$ such that $f X$ dose not belong to $V_{r}$.

We assume that there exsists a point $p$ of $M$ in Case 2). Fix the above point $p$ of $M$. From Lemma 2.2 and (4.4), we get

$$
2\left(r_{i}^{2}+1\right)-\mu\left(2 r_{i}-\alpha\right)=0,
$$


where $r_{i}$ denotes the principal curvature of $X_{i}$.

By the equation (4.6), we see easily that only Case 1) occurs when $M$ is minimal. Using this fact and the Proposition 2.5, we have easily

THEOREM 4.4. Let $M$ be a complete minimal C-Einstein hypersurface of $P^{m}(C)$ such that $b \neq-3$. Then $M$ is congruent to $M_{p, q}^{c}$ for some $p, q$.

THEOREM 4.5. Let $M$ be a complete C-Einstein hypersurface of $P^{m}(C)$ with $m \geqq 3$. If $b \neq-3$ and $a+b \geqq 2(m-1)$ at each point of $M$, then $M$ is congruent to $M_{p, q}^{c}$ for some $p, q$.

Proof. Let $r, r^{\prime}$ be the two real roots of (4.4). We only consider the following case by Lemma 4.3 and Lemma 4.4 :

For any point $p$ of $M$, the tangent space $T_{p} M$ at $p$ can be written as $T_{p} M=V_{\alpha} \oplus V_{r} \oplus V_{r^{\prime}}$ (direct sum), where $\operatorname{dim} V_{\alpha}=1, \quad r \neq r^{\prime}$ and $\operatorname{dim} V_{r}=s$ $(0 \leqq s \leqq 2 m-2)$.

From (4.5), the mean curvature $\mu$ and $\alpha$ have the same sign. If there exists a P.C. vector $X \in V_{r}$ such that $f X \notin V_{r}$, then by (4.6) we have $\mu r=$ $2\left(r^{2}+1\right)+\mu \alpha$. Similarly we get the same equation for $r^{\prime}$. We see that $\mu, r$, and $r^{\prime}$ are non-zero and have the same sign. By the definition of $\mu$, we get

$$
\mu=\operatorname{trace} H=\alpha+\mu+(s-1) r+(2 m-3-s) r^{\prime},
$$

because $r+r^{\prime}=\mu$.

We have $s=1$ and $2 m-3=s$. This is a contradiction for $m \geqq 3$. Then $V_{r}$ and $V_{r^{\prime}}$ are invariant under $f$. This completes the proof by Proposition 2.5.

REMARK 1. We can consider the following special case of Case 2).

Case $2^{\prime}$ )

$$
f X \in V_{r} \quad \text { for any } \quad X \in V_{r} .
$$

Using the compatible submersion $(\bar{M}, M, \pi)$ in Case $\left.2^{\prime}\right)$, the second fundamental tensor of $\bar{M}$ has four principal curvatures whose multiplicities are $1,1, n-1$ and $n-1$. In this case if all the principal curvatures of $M$ are constant, then so are the principal curvatures of $\bar{M}$. The hypersurfaces $\bar{M}$ of $S^{m-1}$ with the above condition have been determined by $\mathrm{R}$. Takagi [5].

REMARK 2. Through an Einstein space is a $C$-Einstein space with $b=0$, there exists no such hypersurface in the class of $M_{p, q}^{c}$ (cf. Proposition 5.5).

\section{$\S 5$. The real hypersurfaces satisfying certain conditions.}

We consider the compatible submersion $(\bar{M}, M, \pi)$. Using the Co-Gauss and the Co-Codazzi equations for this submersion (cf. [1], p. 31), we have easily the following:

LEMMA 5.1. Let $M$ be a real hypersurface of $P^{m}(C)$ and $(\bar{M}, M, \pi)$ a compatible submersion with the Hopf-fibration $\tilde{\pi}$. If $\bar{M}$ is a locally symmetric space, 
then $M$ satisfies

$$
\begin{aligned}
& f H U=0, \\
& f \cdot R=0,
\end{aligned}
$$

where - means that $f$ operates on $R$ as a derivation, i.e., for any vector fields $X, Y, Z$ and $W$ on $M$

$$
\begin{aligned}
g((f \cdot R)(X, Y) Z, W)= & g(R(f X, Y) Z, W)+g(R(X, f Y) Z, W) \\
& +g(R(X, Y) f Z, W)+g(R(X, Y) Z, f W) .
\end{aligned}
$$

In this section we want to discuss the converse problem. Namely the hypersurface $M$ with the condition (5.1) and (5.2) will be determined.

The equation (5.1) implies that $U$ is a P.C. vector with constant principal curvature by (2.3) and Lemma 2.1. So we can apply the results in $\S 2$.

Contracting [5.2) we have

$$
f R_{0}=R_{0} f .
$$

By (1.6) we get for any vectors $X, Y, Z$ and $W$ on $M$

$$
\begin{aligned}
(f \cdot R)(X, Y, Z, W)= & g(H Y, Z) g(H f X, W)-g(H f X, Z) g(H Y, W) \\
& +g(H f Y, Z) g(H X, W)-g(H X, Z) g(H f Y, W) \\
& +g(H Y, f Z) g(H X, W)-g(H X, f Z) g(H Y, W) \\
& +g(H Y, Z) g(H X, f W)-g(H X, Z) g(H Y, f W) .
\end{aligned}
$$

So we have by $(5.2)$

$$
\begin{gathered}
g(H Y, Z) g((H f-f H) X, W)+g(H X, W) g((H f-f H) Y, Z) \\
-g(H Y, W) g((H f-f H) X, Z)-g(H X, Z) g((H f-f H) Y, W)=0 .
\end{gathered}
$$

Similarly the equation (5.3) is equivalent to

$$
\mu(H f-f H) X-\left(H^{2} f-f H^{2}\right) X=0 .
$$

LEMMA 5.2. Let $M$ be a real hypersurface of $P^{m}(C)$ with $m \geqq 3$ satisfying (5.1) and (5.3). If $\alpha=g(H U, U)=0$ at some point $p$ of $M$, there exists a P.C. vector $X \in V_{r}$ such that $g(X, U)=0$ and $f X \in V_{r}$.

Proof. We remarked that $f X$ is also a P.C.-vector if $X$ is a P.C. vector (see $\S 2)$. Take the orthonormal basis $\left\{U, X_{a}, f X_{a},(a=1, \cdots, m-1)\right\}$ consisting of P.C. vectors and denote their principal curvatures by $\alpha, r_{a}, 1 / r_{a}$ respectively, because of Lemma 2.2. Suppose that $r_{a} \neq 1 / r_{a}$, for all $a=1, \cdots, m-1$. In (5.6), replacing $X$ by $X_{i}$, we get 


$$
\left(r_{a}-1 / r_{a}\right)\left(r_{a}+1 / r_{a}-\mu\right)=0 .
$$

It follows $r_{a}+1 / r_{a}=\mu$. On the other hand, we have

$$
\begin{aligned}
\mu & =g(H U, U)+\sum_{a=1}^{m-1} g\left(H X_{a}, X_{a}\right)+\sum_{a=1}^{m-1} g\left(H f X_{a}, f X_{a}\right) \\
& =\sum_{a=1}^{m-1}\left(r_{a}+1 / r_{a}\right)=(m-1) \mu .
\end{aligned}
$$

We have $\mu=0$, which is a contradiction.

LEMMA 5.3. Under the assumptions of Lemma 5.2, the principal curvature of $f X_{a}$ is equal to that of $X_{a}(a=1, \cdots, m-1)$.

Proof. There exists a P.C. vector $X$ with principal curvature $\beta$ such that $\beta^{2}=1$ because of Lemma 5.2, If we take any P.C. vector $X_{a}$ with principal curvature $r_{a}$, then from (5.5), we have

$$
\beta\left(1 / r_{a}-r_{a}\right)\left(g(X, W) g\left(X_{a}, Z\right)-g(X, Z) g\left(X_{a}, W\right)\right)=0,
$$

where $Z$ and $W$ are any vectors on $M$. It follows that $r_{a}=1 / r_{a}$. When $\alpha \neq 0$, replacing $Y$ and $Z$ by $U$ in (5.5), we see that $f$ and $H$ are commutative.

With the above fact and the above lemmas, we have

THEOREM 5.4. Let $M$ be a complete real hypersurface of $P^{m}(C)(m \geqq 3)$. If $M$ satisfies (5.1) and (5.2), then $M$ is congruent to $M_{p, q}^{c}$.

As a final remark, we will show that in $P^{m}(C)$ that there exists no real hypersurface with parallel Ricci tensor in the class of $M_{p, q}^{c}$. Assume that there exists a hypersurface $M_{p, q}^{c}$ with parallel Ricci tensor for some $p, q$. Since $U$ is a P.C. vector with constant principal curvature, using Theorem 0 and (3.1), we have $2 f H+(\mu-\alpha) f=0$, where $\mu=$ trace $H$. Multiplying this equation by $f$ and contracting, we get $\mu=\alpha$. Consequently, $M_{p, q}^{c}$ has the parallel second fundamental tensor. It follows from (3.1) again that $f$ vanishes identically. This is a contradiction.

Using Theorem 4.5 and the above fact, we have

Proposition 5.5. There exists no Einstein hypersurface of $P^{m}(C)(m \geqq 3)$ with scalar curvature $\geqq 2(m-1)(2 m-1)$.

\section{References}

[1] S. Ishihara and M. Konishi, Differential geometry of fibred space, Publ. S.G.G. 8, 1973.

[2] H. B. Lawson, Rigidity theorems in rank-1 symmetric spaces, J. Differential Geometry, 4 (1970), 349-357.

[3] M. Okumura, On the real hypersurfaces of complex projective space, to appear.

[4] J. Ryan, Homogeneity and some curvature conditions for hypersurfaces, Tôhoku Math. J., 1969, 363-388. 
[5] R. Takagi, A class of hypersurfaces with constant principal curvature in a sphere, to appear.

[6] R. Takagi, Real hypersurfaces in a complex projective space with constant principal curvatures, J. Math. Soc. Japan, 27 (1975), 43-53.

[7] Y. Tashiro and S. Tachibana, On Fubinian and C-Fubinian manifolds, Kōkai Math. Semi. Rep., 1963, 176-183.

\section{Yoshiaki MAEDA}

Department of Mathematics Tokyo Metropolitan University

Fukazawa, Setagaya-ku

Tokyo, Japan 